\title{
Ensemble fuzzy models in personalized medicine: application to vasopressors administration
}

\author{
Cátia Salgado ${ }^{a, *}$, Susana M. Vieira ${ }^{\mathrm{a}}$, Luis F. Mendonça ${ }^{\mathrm{a}, \mathrm{b}}$, \\ Stan Finkelstein ${ }^{c, d}$, João M. C. Sousa ${ }^{a}$ \\ ${ }^{a}$ LAETA, IDMEC, Instituto Superior Técnico, Universidade de Lisboa, \\ 1049-001 Lisboa, Portugal \\ ${ }^{b}$ Escola Superior Náutica Infante D. Henrique \\ Department of Marine Engineering, Paço d'Arcos, Portugal \\ ${ }^{c}$ Massachusetts Institute of Technology, Engineering Systems Division, \\ Cambridge, MA, USA \\ ${ }^{d}$ Massachusetts Institute of Technology, Harvard-MIT Division of Health Sciences 83 \\ Technology, Cambridge, MA, USA
}

\begin{abstract}
Vasopressors administration in intensive care units is a risky surgical procedure that can be associated with infections, especially if done urgently such as in the case of unexpected systemic shock. The early prediction of a patient's transition to vasopressor dependence could improve overall outcomes associated with the procedure. Personalized medicine in the ICU encompasses the customization of healthcare on the level of individual patients, with diagnostic tests, monitoring interventions and treatments being fitted to the individual rather than the "average" patient. In this scope, this paper proposes an ensemble fuzzy modeling approach to a classification problem based on subgroups of patients identified by individual characteristics. A fuzzy c-means clustering algorithm was implemented to find subgroups of patients and each subgroup was used to develop a fuzzy model. The final classification of the ensemble fuzzy approach is obtained using two output selection criteria: an a priori decision criterion based on the distance from the clusters centers to the patients' characteristics, and an a posteriori decision criterion based on the uncertainty of the model output. The performance of the proposed approach is investigated using a real world clinical database
\end{abstract}

\footnotetext{
${ }^{*}$ Corresponding author

Email address: catiasalgado@live.com (Cátia Salgado)
}

Preprint submitted to Engineering Applications of Artificial Intelligence October 13, 2015 
and nine benchmark datasets. The ensemble fuzzy model approach performs better than the single model for the prediction of vasopressors administration in the ICU, being the a posteriori approach the best performer, with an average AUC of 0.85 , showing this way the advantage of a personalized approach for patient care in the ICU.

Keywords: Fuzzy modeling, fuzzy clustering, ensemble modeling, vasopressors administration, personalized medicine

\section{Introduction}

Vasopressors drugs are used to contract blood vessels so as to increase blood pressure in critically ill patients. The procedure of vasopressors administration is risky, since the catheter insertion involves a surgical procedure that can be associated with infections. These complications are increased when the procedure is done urgently such as in the case of unexpected systemic shock [1]. If information is available about which patients are going to need vasopressors beforehand, clinicians will have enough time to safely initiate the central line insertion protocol, prior to the moment of need of vasopressors. Being able to predict within a certain time window a patient's transition to vasopressor dependence will likely improve the outcome of ICU patients, either by reducing the number of times this procedure is implemented or by reducing the risk of infection when the procedure is done.

Previous studies have explored the classification of ICU patients on vasopressors. In [2], arterial blood pressure and heart rate are taken as inputs to a fuzzy-logic based algorithm that generates a "vasopressor advisability index'. Following the challenge from PhysioNet and Computers in Cardiology proposed in [3], several algorithms were developed to predict, within a certain time window, which ICU patients would experience an acute hypotensive episode. In [4], a multimodel approach is tested for predicting the risk of death in septic shock patients. Two models were created in parallel, one highlighting sensitivity, and the other highlighting specificity. In [5] and [6], different approaches combining fuzzy modeling with feature selection were used to classify requirements for vasopressors in septic shock patients, focusing exclusively on two disease-based subsets of patients - pancreatitis and pneumonia. As pointed out by the authors, the fact that different features were selected to classify the different groups is an indicator of the relevance of patient specific models in predictive modeling. In fact, several literature 
already exists describing predictive modeling across a range of applications in the care of the critically ill, however, much of this modeling has taken the approach of focusing on the general population, i.e., the "average" patient. Two main reasons can be attributed to this tendency: first it is clear that using a heterogeneous group of patients allows the maximization of external validity of the findings and second, there is a lack of data to support the application of model techniques to smaller subsets. However, as the amount of data increases, the tendency for general population modeling decreases, especially because predictive models developed using this approach often perform poorly when applied to specific subsets of patients sharing common characteristics [7]. In response to treatment, critically ill patients have substantial individual variation between them, and what works at one time might not work later in the same patient [8]. Personalized medicine encompasses the customization of healthcare on the level of individual patients characteristics, with medical decisions, practices and products being fitted to the individual rather than the "average" patient [9]. Hence, the near future is likely to see a new era of personalized medicine in the treatment of the critically ill [10].

Ensemble learning is an important branch in the research area of machine learning $[11,12]$. Ensemble models have been applied to a wide range of complex real problems $[13,14,15,16]$ and several authors have demonstrated the advantages of using this type of approach, in weather forecasts $[17,18]$, credit risk $[19,20]$, fault diagnosis [21] and clinical care $[4,22]$. The rationale behind ensemble classification is the creation of many classifiers such that the combination or selection of their output improves the performance of the single classifier [23]. In literature, distinct types of architectures topology and decision criteria strategies have been proposed and discussed. Serial architectures use a primary classifier to evaluate a given instance, and when that classifier is not able to classify some new pattern, another one is trained in order to be accurate on the errors of the previous. A third and fourth classifier can be added, and so on. Parallel architectures use the combination of the output of several models trained in parallel to obtain the final decision. Various methods have been proposed for combining classifiers, where relevant triggering or aggregating mechanisms are defined to activate or define a suitable interaction between them [23, 24, 25]. These include majority voting [13], bayesian voting [16], bagging [26], boosting [27, 28] and classifier selection $[29,30]$.

In this paper, we propose an ensemble model approach for personalized medicine based on fuzzy clustering and fuzzy modeling. Unsupervised fuzzy 
clustering is used to identify similar patients and generate different data divisions. Each division is used to train individual models, resulting in a specific model for each group of similar patients. Thus, in contrast to previous approaches by $[4,5,6]$, submodels are developed for specific groups of patients identified by fuzzy clustering. Two selection decision criteria are proposed to obtain the final classification: an a priori decision based on data properties, i.e., distance from the clusters centers to the patient characteristics; and an a posteriori decision approach based on the uncertainty of the model output. The goal is to see if larger predictive performance can be achieved using an ensemble model, as compared to a single model approach. This hypothesis is tested on a real problem for the prediction of vasopressors dependency in ICU patients and in nine benchmarks datasets from the UCI benchmark repository [31].

The outline of the paper is as follows: in Section 2, the basis for the clustering methodology is introduced, followed by the description of fuzzy modeling and model performance measures. In Section 3, the two proposed ensemble approaches are described. Section 4 presents a description of the benchmark and vasopressors datasets that are used to evaluate the performance of the models, followed by a discussion of results in Section 5. Conclusions and future work are drawn in Section 6.

\section{Methods}

\subsection{Clustering}

Clustering is an unsupervised learning task that aims at decomposing a given set of samples into subgroups based on data similarity [32]. In this work, fuzzy clustering is used to divide ICU patients in subgroups.

Fuzzy clustering allows each sample to belong to more than one cluster with different membership degrees. Fuzzy c-means (FCM) is the most popular objective function based fuzzy clustering algorithm. It was first developed by Dunn [33] and improved by Bezdek [34]. In FCM, the clustering of a dataset $X$ containing $N$ samples, into $C$ clusters is defined to be optimal when it minimizes the c-means functional formulated as:

$$
J_{f}\left(X, U_{f}, V\right)=\sum_{i=1}^{C} \sum_{j=1}^{N} \mu_{i j}^{m} d_{i j}^{2}\left(x_{j}, v_{i}\right)
$$


where $U_{f}=\left[\mu_{i j}\right]$ is a $C \times N$ matrix, called a fuzzy partition matrix, $V=$ $\left[v_{1}, v_{2}, \ldots, v_{C}\right], v_{i} \in R^{n}$ is a vector of cluster prototypes (centers), $m \in[1, \infty[$ is the weighting exponent which determines the clustering degree of fuzziness, $d_{i j}$ is the distance between data point $x_{j}$ and cluster center $v_{i}$, and is a squared inner-product distance given by (2), where $A$ is a norm-inducing matrix:

$$
d_{i j}^{2}\left(x_{j}, v_{i}\right)=\left(x_{j}-v_{i}\right)^{T} A\left(x_{j}-v_{i}\right)
$$

Since the FCM algorithm requires the definition of $C$, [34], a validity measure called separation index, $S$, was used to find the most adequate number of clusters in the data [35]. This measure uses a minimum-distance separation for partition validity, and is defined as:

$$
S=\frac{\sum_{i=1}^{C} \sum_{j=1}^{N}\left(\mu_{i j}\right)^{2}\left\|x_{j}-v_{i}\right\|^{2}}{N_{\text {min } i, k}\left\|v_{k}-v_{i}\right\|^{2}}
$$

Other measures, such as partition index [36], partition entropy [37] and partition coefficient [37] were also used for comparison purposes. However, for the sake of simplicity and as no significant differences were found, only the results for the separation index validation measure are shown. For the clustering step, Matlab Fuzzy Clustering and Matlab Data Analysis toolboxes were used [38].

\subsection{Fuzzy modeling}

Fuzzy modeling is a tool that allows approximation of nonlinear systems when there is little or no previous knowledge of the problem to be modeled [39]. This approach provides a transparent, non-crisp model, and allows linguistic interpretations in the form of rules and logical connectives, which are particularly useful in clinical scenarios. These rules are used to establish relations between the defined features in order to derive a model. A fuzzy classifier contains a rule base consisting of a set of fuzzy if-then rules together with a fuzzy inference mechanism. The model ultimately classifies each instance of the dataset as pertaining with a certain degree to one of the possible classes defined for the specific problem being modeled.

In this work, Takagi-Sugeno (TS) fuzzy models [40] were used to develop the patient subgroups classifiers. This type of fuzzy modeling consists of input-output rules of the type:

$$
\begin{array}{r}
R_{i}: \text { If } x_{1} \text { is } A_{i 1} \text { and } \ldots \text { and } x_{n} \text { is } A_{i n} \\
\text { then } y_{i}=a_{i 1} x_{1}+\ldots+a_{i n} x_{n}+b_{i},
\end{array}
$$


where $i=1,2, \ldots, K$ denotes the rule number in the rule base. $R_{i}$ is the $i$ th rule, $y_{i}$ is the output of the $i$ th rule, $A_{i 1}, \ldots, A_{i n}$ are the antecedent fuzzy sets, $a_{i}=\left[a_{i 1}, \ldots, a_{i n}\right]$ is a parameter vector, $b_{i}$ is a scalar offset and $x=$ $\left[x_{1}, x_{2}, \ldots, x_{n}\right]^{T}$ is the input vector, where $n$ is the number of input variables. The degree of activation of the $i$ th rule is given by:

$$
\beta_{i}(x)=\prod_{j=1}^{n} \mu_{A_{i j}}(\mathbf{x}),
$$

where $\mu_{A_{i j}}: R \rightarrow[0,1]$ is the membership function of the fuzzy set $A_{i j}$ in the antecedent of $R_{i}$. The model output is determined through the weighted average of the individual rule outputs. The number of rules $K$ and the antecedent fuzzy sets $A_{i j}$ are determined using fuzzy clustering in the product space of the input and output variables [41], with $A_{i j}$ being approximated by Gaussian functions. In this case, the clustering method used is the FCM. The consequent parameters of $y_{i}$, namely $b_{i}$ and $a_{i}$, are obtained using a weighted ordinary least-square estimate.

A threshold $\delta$ is required to turn the continuous output $y \in[0,1]$ into the binary output $o \in\{0,1\}$. Thus, the predicted output is 1 if $y \geq \delta$ and 0 if $y<\delta$.

\subsection{Model assessment}

In describing the performance of binary classifiers, the accuracy of classification cannot be considered alone [42]. Especially in medical applications, this measure shows limitations, where the positive class (disease-positive) is often underrepresented and misclassifications in this class will not have great impact in the accuracy value. From the clinical perspective, achieving the highest sensitivity and specificity simultaneously is often desirable [43]. In medical diagnosis and in the machine learning community, one of the methods for combining sensitivity and specificity measures into the evaluation task is the analysis of the area under the receiver-operator characteristics (ROC) curve (AUC), a graphical plot which illustrates the performance of a binary classifier system as its discrimination threshold is varied [44]. A value of 0.50 is achieved through random predictions and 1 represents a perfect discrimination.

In this work, the measures used to assess the quality of the obtained classifiers were: AUC; specificity, which represents the ratio of true negatives that were identified in all the negative class samples; sensitivity, which 
represents the ratio of true positives that were identified in all the positive class samples; and the accuracy, which consists on the proportion of true classifications over the total number of observations.

Results were obtained using $60 \%$ of the data for the unsupervised clustering and training model and the other $40 \%$ for test. A 10 -fold cross-validation step was applied for testing all datasets. To reduce variability, 20 rounds of this division process were performed always using different partitions, and results were averaged over the rounds.

The threshold $\delta$ selected to turn the continuous output into a binary classification is determined for each model. This threshold is optimized in order to maximize the AUC of the training set in each round of the cross validation step.

\section{Proposed ensemble fuzzy modeling}

Typically, ensemble methods use random sampling for the creation of specific training subsets over the entire feature space. In order to better deal with data-point specific characteristics, we propose a structured division of data to model individual classifiers which is in contrast to random subset generation. Unsupervised FCM was implemented to obtain different clusters representing patients with similar data and a fuzzy model was developed for each cluster, meaning that the number of models in the ensemble is equal to the number of clusters. In order to select which model provides the classification, two decision criteria were used: an a priori decision, where the decision is performed before the assessment of the model; and an a posteriori decision, where the decision is performed after the evaluation of the model. We also developed a single model using the complete train data without patients subgroup division, for comparison purposes. The proposed decision criteria are presented in the next subsections.

\subsection{A priori decision}

The a priori decision criterion is based on cluster similarity, i.e, on samples characteristics similarity. The FCM algorithm was used to obtain $C$ clusters on training data, where $C$ is determined using the separation index described in (3). The distance $D_{i}$ between data points $x_{j}$ and the clusters centers $v_{i}$ is given by:

$$
D_{i}=\left|x_{j}-v_{i}\right|
$$


where $i=1,2, \ldots, C$. Then, the minimum distance $D_{i}$ is selected, as well as the correspondent cluster as:

$$
l=\arg \min \left(D_{i}\right)
$$

being $l$ the index that corresponds to the minimum value of $D_{i}$. The model that classifies the data point $x_{j}$ is the one that was trained with data from the selected cluster. Figure 1 shows a schematic representation of the ensemble fuzzy model approach using the a priori criterion, where $M_{1}, \ldots, M_{C}$ represent fuzzy models and $M_{l}$ the resulting model of $l$.

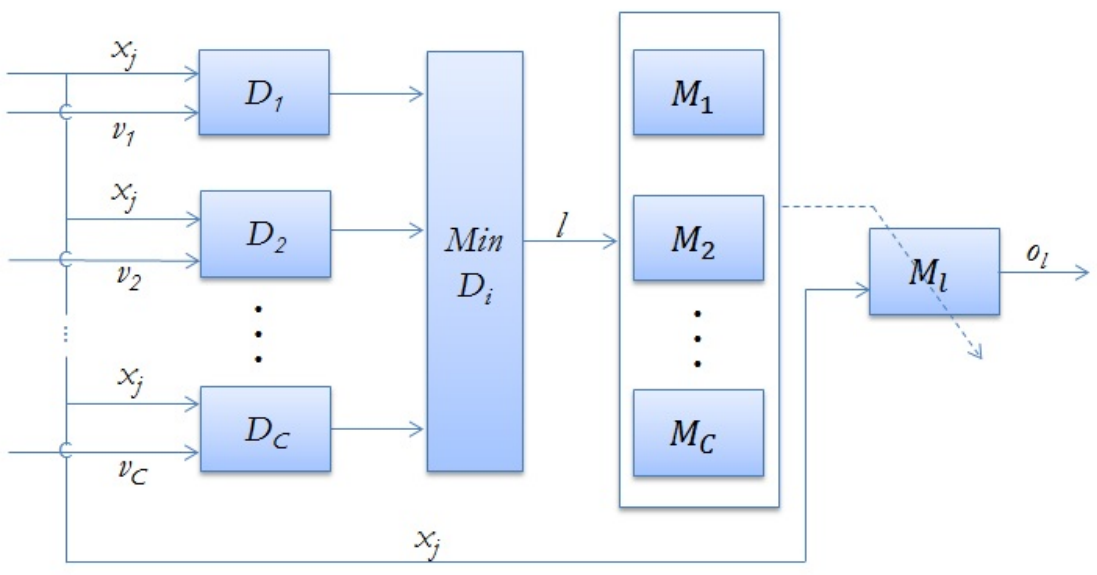

Figure 1: Ensemble fuzzy model scheme with a priori decision based on cluster similarity.

\subsection{A posteriori decision}

In this case, the ensemble fuzzy model approach implements an a posteriori decision, which is based on the uncertainty associated with the predicted outcome and the threshold of each model $\delta_{i}$. The FCM algorithm was applied and $C$ clusters were obtained. For each cluster the respective model was constructed in a total of $C$ models. The difference between the output $o_{i}$ and the threshold $\delta_{i}$ of each model was computed as:

$$
r_{i}=\left|\delta_{i}-o_{i}\right|
$$


where $i=1, \ldots, C$. The model selected to classify the data point is the one that corresponds to the maximum difference $r_{t}$ given by:

$$
r_{t}=\max \left(r_{i}\right)
$$

This is based on the hypothesis that a predicted value distant from the threshold is more certainly assigned to one of the classes than a value closer to the threshold.

The ensemble fuzzy model scheme of this decision criterion is defined in Figure 2.

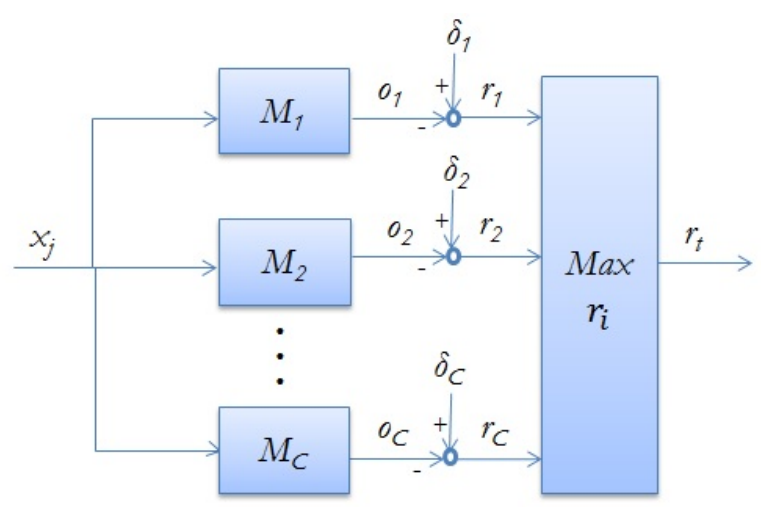

Figure 2: Ensemble fuzzy model scheme with a posteriori decision based on model output uncertainty.

\section{Datasets description}

\subsection{Benchmark}

To validate the proposed approach in a general way rather than just for the vasopressors healthcare application, benchmark datasets were used.

The single and ensemble model approaches were applied to nine standard machine learning datasets from the UCI collection [31], which is a repository widely used by researchers in the machine learning field. The characteristics of the datasets are summarized in Table 1. 
Table 1: Benchmarks datasets characteristics.

\begin{tabular}{lccc} 
Databases & Size & $\begin{array}{c}\text { Number of } \\
\text { features }\end{array}$ & $\begin{array}{c}\text { Number of } \\
\text { classes }\end{array}$ \\
\hline WBCO & 699 & 9 & 2 \\
WBCD & 569 & 30 & 2 \\
WBCP & 198 & 32 & 2 \\
Glass & 214 & 9 & 2 \\
Pima & 768 & 8 & 2 \\
Transfusion & 748 & 4 & 2 \\
ILPD & 583 & 8 & 2 \\
Liver & 345 & 6 & 2 \\
Sonar & 208 & 60 & 2 \\
\hline
\end{tabular}

\subsection{MIMIC II}

Multi-parameter Intelligent Monitoring in Intensive Care (MIMIC II) Database is a de-identified publicly available ICU database composed of detailed information of more than 32,000 patients [45]. Patients were admitted between 2001 and 2008 to the Beth Israel Deaconess Medical Center (BIDMC), an academic medical center in Boston with 620 beds, 77 of which are exclusive for critical care. MIMIC II contains information regarding patient demographics, physiological measures, procedures, medications, laboratory tests results, fluid balance and nursing notes, organized into a relational database. This information can be queried or downloaded from the database website. $^{1}$

Two specific groups of patients were selected for the construction of the datasets: pancreatitis and pneunomia. These are two conditions prone to the development of systemic shock that end up requiring the possible use of fluids and vasopressor agents. Groups were constructed based on ICD-9 codes, an hierarchical medical coding system that allows health conditions (sign, symptom or disease) to be associated with an unique code. Inclusion criteria for the dataset included adult patients that had at least one ICD-9 record associated with pancreatitis and/or pneumonia. The most commonly recorded variables for these groups were selected as the features for the classification

\footnotetext{
${ }^{1}$ http://physionet.org/mimic2/
} 
problem, as indicated in Table 2. Variables in bold (1, 3, 4, 19 and 24) represent features with sampling rates around one sample per hour, while the remaining represent those with sampling rates of up to one record per twelve hours. As in [5], the list of vasopressors selected includes phenylepherine (Neosynepherine), norepinepherine (Levophed) and dobutamine (Dobutex).

Table 2: Physiological variables used.

\begin{tabular}{|c|c|}
\hline \# & Variable (units) \\
\hline 1 & HR - Heart Rate (beats/min) \\
\hline 2 & Temperature $(\mathrm{C})$ \\
\hline 3 & $\mathrm{SpO} 2(\%)$ \\
\hline 4 & RR - Respiratory Rate (breaths/min) \\
\hline 5 & GCS Total \\
\hline 6 & Braden Score \\
\hline 7 & Hematocrit (\%) \\
\hline 8 & Platelets (cells/L) \\
\hline 9 & WBC - White Blood Cells (103/mL) \\
\hline 10 & Hemoglobin $(\mathrm{g} / \mathrm{L})$ \\
\hline 11 & RBC - Red Blood Cells (106/mL) \\
\hline 12 & BUN - Blood urea nitrogen $(\mathrm{mg} / \mathrm{dL})$ \\
\hline 13 & Creatinine $(\mathrm{mg} / \mathrm{dL})$ \\
\hline 14 & Glucose $(\mathrm{mg} / \mathrm{dL})$ \\
\hline 15 & Potassium $(\mathrm{mEq} / \mathrm{L})$ \\
\hline 16 & Chloride $(\mathrm{mEq} / \mathrm{L})$ \\
\hline 17 & Sodium $(\mathrm{mEq} / \mathrm{L})$ \\
\hline 18 & Magnesium (mg/dL ) \\
\hline 19 & NBP - Non-invasive blood pressure ( $\mathrm{mmHg}$ ) \\
\hline 20 & NBP Mean $(\mathrm{mmHg})$ \\
\hline 21 & Arterial pH \\
\hline 22 & Arterial Base Excess $(\mathrm{mEq} / \mathrm{L})$ \\
\hline 23 & Lactic Acid $(\mathrm{mg} / \mathrm{dL})$ \\
\hline 24 & UO - Urine Output $(\mathrm{mL})$ \\
\hline
\end{tabular}

Data preprocessing is an important step for the improvement of the quality of data. In this work, missing data was addressed using the last value carried forward method [46, 47, 48]; outliers were removed using the inter- 
quartile range method [49]; normalization used the min-max procedure; and finally data was aligned with a gridding approach based on heart rate sampling frequency [50]. Heart rate was chosen as the template because it is the most frequently measured variable and thus, the one that introduces fewer artifacts in the data [51]. Each input vector is composed of $n$ components, such that $x=\left[x_{1}, x_{2}, \ldots, x_{n}\right]$, where $x_{i}, i=1,2, \ldots, n$, corresponds to the measurement of the variable at each hour. The output constitutes a binary classification with positive value if the patient was on vasopressors and zero if not.

\section{Results and discussion}

\subsection{Benchmark}

The ideal number of clusters was assessed using the separation index as described in (3). Two clusters were selected for WBCO, WBCD, WBCP, Pima, Transfusion and Liver, and three for Glass, indian liver patient dataset (ILPD) and Sonar datasets. The number of rules in each subgroup was two for WBCO, WBCD, WBCP, Pima, Transfusion and Liver, and three for Glass, ILPD and Sonar datasets. The degree of fuzziness $m$ was set to 1.8.

Performance results are shown in Table 3, with best values depicted in bold. Overall, the ensemble model performs better or equal than the single model. The resulting win/tie/loss counts for AUC are shown in the last lines of Table 4. Whenever the ensemble model approach achieves better/worse performance than the single model on any dataset, a win/loss is counted and a marker $\bullet / 0$ is shown. A tie is also counted but no marker is given. The overall win/tie/loss is given at the bottom of the table by comparing all models (single model and ensemble model).

Table 4 shows that the a priori ensemble performed better than the single model on nine out of nine datasets. This difference is statistically significant, with $\mathrm{p}<0.05$. The a posteriori ensemble also performs better than the single model in a large number of datasets (seven out of nine). Relatively to the two types of ensemble approaches, the a priori decision obtains better results (with overall win/tie/loss record of $7 / 1 / 1$ ) when compared with the a posteriori decision (with overall win/tie/loss record of $1 / 1 / 7$ ). 
Table 3: Benchmarks databases results.

\begin{tabular}{|c|c|c|c|c|}
\hline & & Single model & a priori & a posteriori \\
\hline \multirow[t]{4}{*}{ WBCO } & AUC & $0.97 \pm 0.01$ & $0.98 \pm 0.01$ & $0.98 \pm 0.01$ \\
\hline & Accuracy & $0.97 \pm 0.01$ & $0.98 \pm 0.01$ & $0.97 \pm 0.01$ \\
\hline & Sensitivity & $0.97 \pm 0.01$ & $0.97 \pm 0.01$ & $0.96 \pm 0.01$ \\
\hline & Specificity & $0.98 \pm 0.01$ & $0.99 \pm 0.01$ & $0.99 \pm 0.01$ \\
\hline \multirow[t]{4}{*}{ WBCD } & AUC & $0.95 \pm 0.01$ & $0.96 \pm 0.01$ & $0.97 \pm 0.01$ \\
\hline & Accuracy & $0.95 \pm 0.01$ & $0.97 \pm 0.01$ & $0.97 \pm 0.01$ \\
\hline & Sensitivity & $0.96 \pm 0.02$ & $0.98 \pm 0.01$ & $0.97 \pm 0.01$ \\
\hline & Specificity & $0.95 \pm 0.02$ & $0.94 \pm 0.01$ & $0.97 \pm 0.02$ \\
\hline \multirow[t]{4}{*}{ WBCP } & AUC & $0.63 \pm 0.05$ & $0.72 \pm 0.05$ & $0.65 \pm 0.06$ \\
\hline & Accuracy & $0.60 \pm 0.12$ & $0.70 \pm 0.04$ & $0.67 \pm 0.04$ \\
\hline & Sensitivity & $0.58 \pm 0.21$ & $0.69 \pm 0.04$ & $0.68 \pm 0.05$ \\
\hline & Specificity & $0.69 \pm 0.18$ & $0.76 \pm 0.10$ & $0.62 \pm 0.11$ \\
\hline \multirow[t]{4}{*}{ Glass } & AUC & $0.91 \pm 0.04$ & $0.95 \pm 0.02$ & $0.88 \pm 0.04$ \\
\hline & Accuracy & $0.93 \pm 0.03$ & $0.93 \pm 0.02$ & $0.93 \pm 0.03$ \\
\hline & Sensitivity & $0.89 \pm 0.08$ & $0.98 \pm 0.03$ & $0.79 \pm 0.08$ \\
\hline & Specificity & $0.94 \pm 0.03$ & $0.92 \pm 0.03$ & $0.97 \pm 0.03$ \\
\hline \multirow[t]{4}{*}{ Pima } & AUC & $0.76 \pm 0.02$ & $0.79 \pm 0.02$ & $0.77 \pm 0.02$ \\
\hline & Accuracy & $0.76 \pm 0.03$ & $0.78 \pm 0.02$ & $0.76 \pm 0.03$ \\
\hline & Sensitivity & $0.75 \pm 0.07$ & $0.75 \pm 0.05$ & $0.76 \pm 0.07$ \\
\hline & Specificity & $0.78 \pm 0.07$ & $0.82 \pm 0.05$ & $0.77 \pm 0.07$ \\
\hline \multirow[t]{4}{*}{ Tranfusion } & AUC & $0.71 \pm 0.03$ & $0.72 \pm 0.03$ & $0.71 \pm 0.03$ \\
\hline & Accuracy & $0.75 \pm 0.04$ & $0.75 \pm 0.03$ & $0.76 \pm 0.03$ \\
\hline & Sensitivity & $0.79 \pm 0.06$ & $0.78 \pm 0.03$ & $0.80 \pm 0.04$ \\
\hline & Specificity & $0.62 \pm 0.09$ & $0.65 \pm 0.04$ & $0.62 \pm 0.07$ \\
\hline \multirow[t]{4}{*}{ ILPD } & AUC & $0.68 \pm 0.03$ & $0.72 \pm 0.02$ & $0.69 \pm 0.02$ \\
\hline & Accuracy & $0.65 \pm 0.05$ & $0.67 \pm 0.04$ & $0.65 \pm 0.04$ \\
\hline & Sensitivity & $0.59 \pm 0.11$ & $0.60 \pm 0.08$ & $0.60 \pm 0.08$ \\
\hline & Specificity & $0.77 \pm 0.12$ & $0.83 \pm 0.07$ & $0.79 \pm 0.07$ \\
\hline \multirow[t]{4}{*}{ Liver } & AUC & $0.71 \pm 0.03$ & $0.76 \pm 0.03$ & $0.73 \pm 0.03$ \\
\hline & Accuracy & $0.72 \pm 0.03$ & $0.76 \pm 0.03$ & $0.75 \pm 0.03$ \\
\hline & Sensitivity & $0.66 \pm 0.09$ & $0.73 \pm \mathbf{0 . 0 9}$ & $0.60 \pm 0.10$ \\
\hline & Specificity & $0.77 \pm 0.09$ & $0.79 \pm 0.08$ & $0.85 \pm 0.07$ \\
\hline \multirow[t]{4}{*}{ Sonar } & AUC & $0.67 \pm 0.11$ & $0.89 \pm 0.02$ & $0.76 \pm 0.03$ \\
\hline & Accuracy & $0.68 \pm 0.07$ & $0.87 \pm 0.03$ & $0.73 \pm 0.04$ \\
\hline & Sensitivity & $0.66 \pm 0.16$ & $0.91 \pm 0.03$ & $0.88 \pm 0.05$ \\
\hline & Specificity & $0.68 \pm 0.16$ & $0.86 \pm \mathbf{0 . 0 3}$ & $0.63 \pm 0.05$ \\
\hline
\end{tabular}


Table 4: AUC performance of benchmarks databases . •/o indicates whether ensemble approaches are better/worse than the single model.

\begin{tabular}{lccc} 
& Single model & a priori & a posteriori \\
\hline WBCO & $0.97 \pm 0.01$ & $0.98 \pm 0.01 \bullet$ & $0.98 \pm 0.01 \bullet$ \\
WBCD & $0.95 \pm 0.01$ & $0.96 \pm 0.01 \bullet$ & $0.97 \pm 0.01 \bullet$ \\
WBCP & $0.63 \pm 0.05$ & $0.72 \pm 0.05 \bullet$ & $0.65 \pm 0.06 \bullet$ \\
Glass & $0.91 \pm 0.04$ & $0.95 \pm 0.02 \bullet$ & $0.88 \pm 0.04 \circ$ \\
Pima & $0.76 \pm 0.02$ & $0.79 \pm 0.02 \bullet$ & $0.77 \pm 0.02 \bullet$ \\
Tranfusion & $0.71 \pm 0.03$ & $0.72 \pm 0.03 \bullet$ & $0.71 \pm 0.03$ \\
ILPD & $0.68 \pm 0.03$ & $0.72 \pm 0.02 \bullet$ & $0.69 \pm 0.02 \bullet$ \\
Liver & $0.71 \pm 0.03$ & $0.76 \pm 0.03 \bullet$ & $0.73 \pm 0.03 \bullet$ \\
Sonar & $0.67 \pm 0.11$ & $0.89 \pm 0.02 \bullet$ & $0.76 \pm 0.03 \bullet$ \\
\hline Win/Tie/Loss & - & $9 / 0 / 0$ & $7 / 1 / 1$ \\
Overall Win/Tie/Loss & $0 / 0 / 9$ & $7 / 1 / 1$ & $1 / 1 / 7$ \\
\hline
\end{tabular}

We compared the accuracy of the proposed models with previous benchmark results found in literature. The proposed ensemble fuzzy models performed better than five other approaches in the classification of benchmark data: in [52], Support Vector Machines were used to classify the Glass dataset with an accuracy of 71-73.8\%; in [53], the nearest mean classifier with boosting achieved $74 \%$ accuracy in the classification of Pima; the global tree optimization classified Liver with an accuracy of $60.6-68.7 \%$ in [54]; Fuzzy AIRS with Liver achieved $70 \%$ [55] and an accuracy of $76 \%$ was found using MML Oblique Trees in [56]. Similar results were found for WBCO, Transfusion and ILPD, using neural networks with random weights in [57], with 94-98\%, 73-84\% and 59-67\% accuracy respectively; and WBCD with Fuzzy AIRS in [55] also presented similar results (94.7-98.5\% accuracy). The proposed approach performed worse in the classification of WBCP and Liver than [58] (93\% with neural networks) and [55] (83\% with Fuzzy AIRS) respectively.

\subsection{MIMIC II}

The ideal number of clusters was assessed using the separation index in (3), for a sequence of cluster numbers ranging from 2 to 10 . The degree of fuzziness $m$ was also 1.8. Two different datasets were built using the records in the whole training set: one without feature selection and the other using the 5 most frequently sampled features (heart rate, $\mathrm{SpO} 2$, respiratory rate, 
NBP and urine output). The reasoning behind this choice relates to the fact that using only the most frequently sampled features, better results are likely to be obtained, as less errors associated with the interpolation step are introduced. Moreover, this set of variables are easily acquired and in most cases readily accessible at admission.

Figure 3 shows a monotonic curve of the separation index results for the two datasets. The reduced dataset was divided into four clusters since this is a

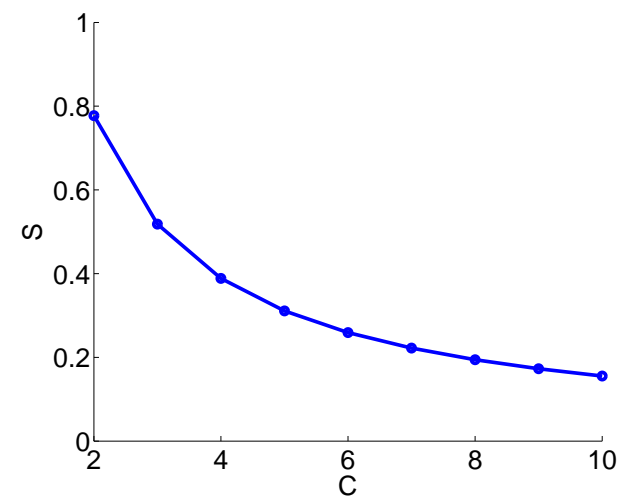

(a)

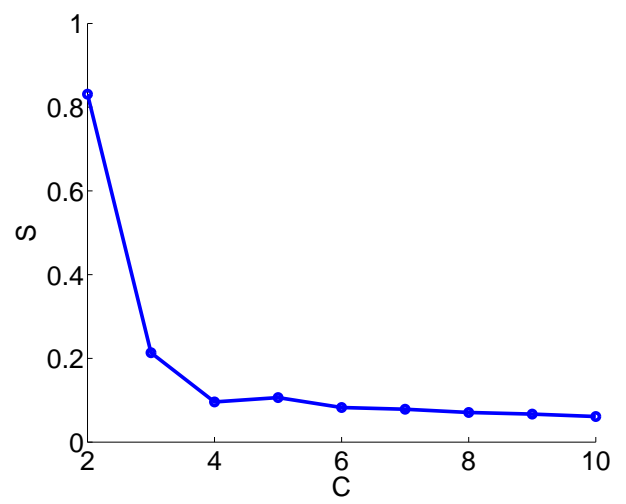

(b)

Figure 3: Separation index using: (a) the full dataset; and (b) the most frequently sampled variables.

clear local minimum of $S$. For the sake of simplicity and interpretability, this number was preferred over the other local minima, and it was also selected for the full dataset. The number of rules in each subgroup was 4. As shown in Figure 4, the number of patients in each cluster was found to be evenly spread throughout all clusters (around 300 patients each), for the reduced dataset. Moreover, both classes of patients - patients in vasopressors and patients that did not receive the treatment - were distributed with similar proportions in the groups, meaning that the distribution of patients across groups does not reflect an even distribution of outcomes, but rather a distribution based on physiological trends. Performance results in Table 5 show that the models are able to take advantage of this division, to improve classification results as compared to a single model approach.

In order to determine which features to use, the single model approach was performed for both datasets. Table 5 shows that the single model developed upon the reduced dataset has significantly lower performance than 


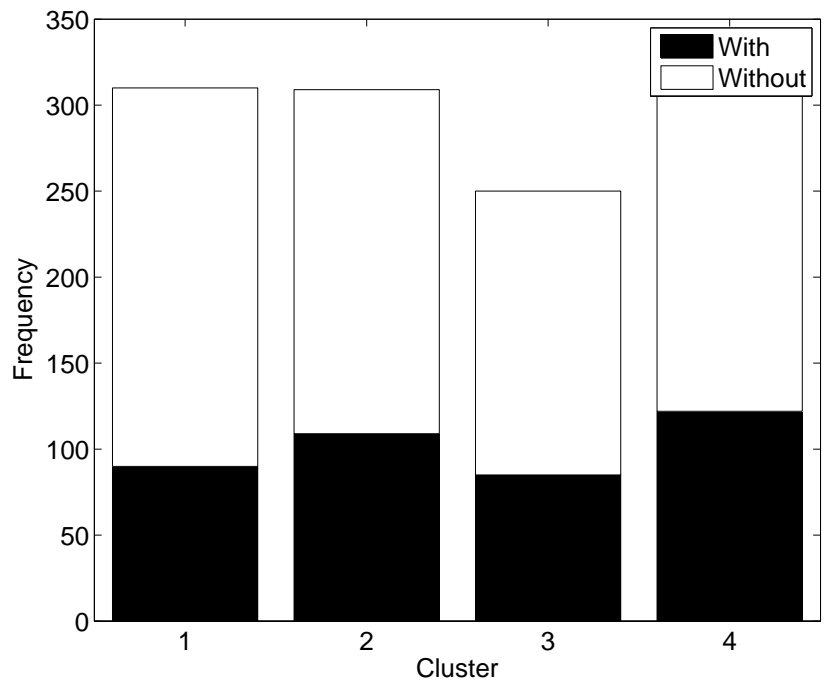

Figure 4: Patients in each of the four clusters with and without vasopressors, using the reduced dataset.

the single model using the whole feature set. Since no feature selection step was performed, the lower performance can mean that information was lost as some important variables were left out.

Overall results in Table 5 show that: i) the AUC of both ensemble approaches is larger than the single model; ii) unlike benchmark results, the $a$ posteriori approach returned significantly better classification performance than the others, with a better proportion of patients that were correctly classified as being administrated with vasopressors, and a smaller false alarm rate, i.e, more patients not being administrated with vasopressors also correctly classified as such. Benchmark data is usually complete, consistent and time stamped, with a high degree of quality. Since the a priori decision relies on the distance to the cluster centers, it is more based on the characteristics of the data, which can explain why it is preferred for such datasets. On the other hand, since the real data is more incomplete and difficult to cluster, the criteria based on the uncertainty of the model provides better results. In addition, while using the reduced set to build a general model returned worse results, using the same information to build the ensemble lead to good results in comparison.

Improvements in the performance were notable compared to similar works. 
In particular, comparing to the study of Fialho et al. [6], where the same cohort of patients was used to predict vasopressors dependence, an increase of 0.06 in AUC, sensitivity and specificity was achieved using the a posteriori decision criterion.

Table 5: Mean and standard deviation of the results obtained after 20 runs for the single and ensemble model approaches. The best AUC, accuracy, sensitivity and specificity for each dataset are highlighted in bold.

\begin{tabular}{ccccc} 
& & Single model & a priori & a posteriori \\
\hline \multirow{4}{*}{24} & AUC & $0.80 \pm 0.03$ & $0.81 \pm 0.02$ & $\mathbf{0 . 8 5} \pm \mathbf{0 . 0 1}$ \\
features & Accuracy & $0.80 \pm 0.03$ & $0.80 \pm 0.02$ & $\mathbf{0 . 8 5} \pm \mathbf{0 . 0 1}$ \\
& Sensitivity & $0.80 \pm 0.03$ & $0.82 \pm 0.01$ & $\mathbf{0 . 8 5} \pm \mathbf{0 . 0 0}$ \\
& Specificity & $0.81 \pm 0.04$ & $0.80 \pm 0.00$ & $\mathbf{0 . 8 4} \pm \mathbf{0 . 0 1}$ \\
\hline \multirow{5}{*}{5} & AUC & $0.71 \pm 0.01$ & $0.72 \pm 0.00$ & $\mathbf{0 . 7 5} \pm \mathbf{0 . 0 1}$ \\
features & Accuracy & $0.72 \pm 0.03$ & $0.72 \pm 0.02$ & $\mathbf{0 . 7 9} \pm \mathbf{0 . 0 1}$ \\
& Sensitivity & $0.72 \pm 0.03$ & $0.72 \pm 0.02$ & $\mathbf{0 . 7 8} \pm \mathbf{0 . 0 2}$ \\
& Specificity & $\mathbf{0 . 7 1} \pm \mathbf{0 . 0 2}$ & $0.70 \pm 0.02$ & $0.70 \pm 0.02$ \\
\hline
\end{tabular}

An example of the membership functions obtained for each variable is depicted in Figure 5. These functions were obtained after simplification and reduction of the fuzzy model, by merging similar fuzzy sets and removing fuzzy sets similar to the universal set, based on the fuzzy clustering toolbox proposed by Babuška et al. (more details can be found in [59]). Similar membership functions were merged in 5 out of 5 variables (rule 2 and rule 3 ), and the universal set was removed in the respiratory rate and SpO2 (rule 2). 

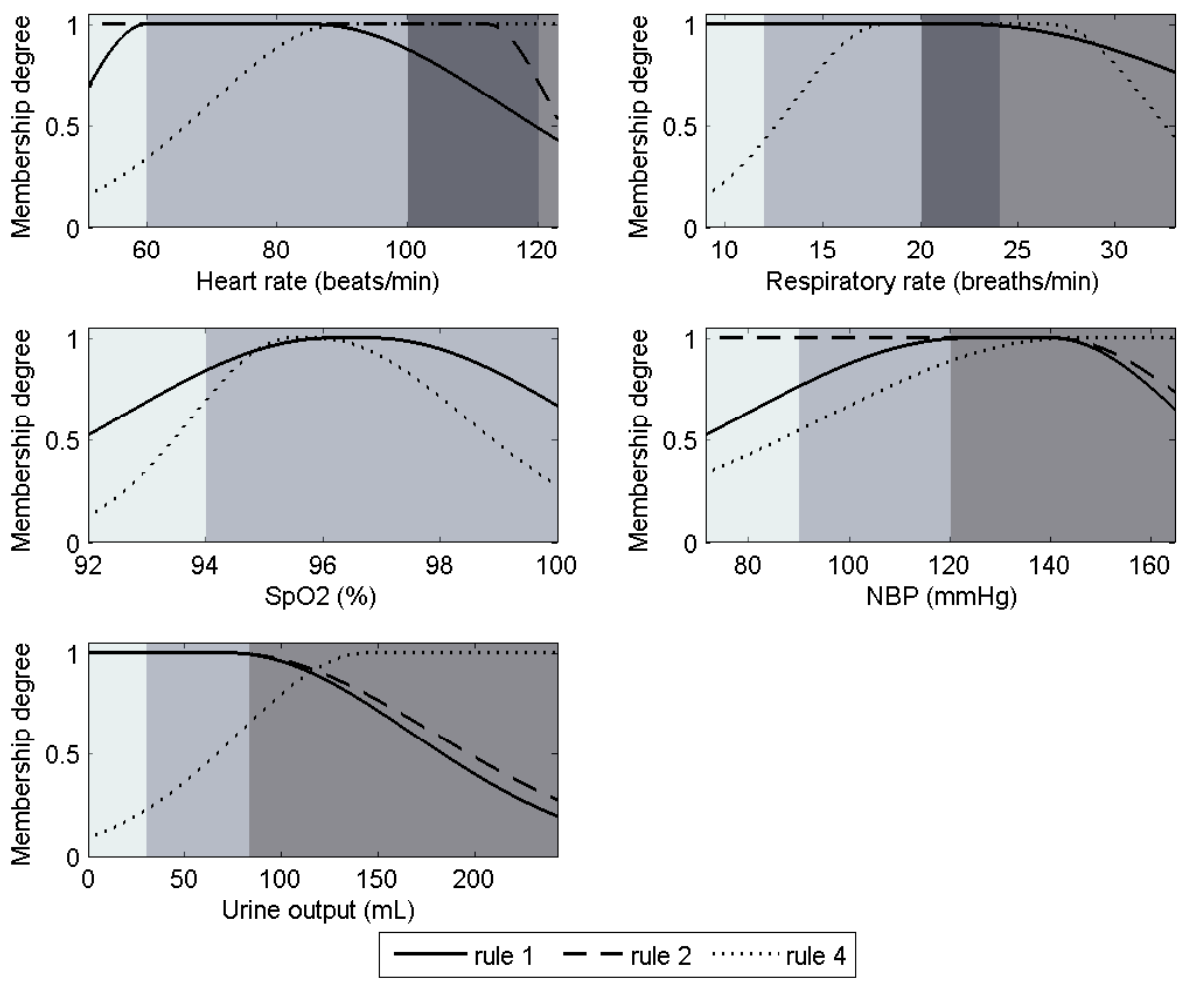

Figure 5: Membership functions obtained for assessing vasopressors dependency, in the reduced dataset. Each line corresponds to a cluster rule. Lower and upper bounds accepted for low, normal and high values are also depicted in light blue, blue and grey, respectively.

Light blue, blue and grey regions corresponding respectively to ranges of low, normal and high values of each variable, are represented in Figure 5 to support further linguistic interpretation of the rules. The accepted normal ranges of each variable were defined as 60 to 120 beats/min for heart rate, 12 to 24 breaths/min for respiratory rate, 94 to $100 \%$ for SpO2, 90 to 120 $\mathrm{mmHg}$ for NBP and 30 to $83 \mathrm{~mL} /$ hour for urine output.

The model created consists of non-linear combinations and therefore we cannot investigate any of the variables separately from the others. We were able to extract four distinct rules that can constitute a first assessment of vasopressors dependency in the ICU. The if-then rules are as follows:

1. If $\mathrm{HR}$ is slightly low or normal and $\mathrm{RR}$ is low or normal and $\mathrm{SpO} 2$ is normal and NBP is slightly high and UO is slightly low or normal, 
then $y_{1}=0.476 \mathrm{HR}+0.046 \mathrm{RR}+1.20 \mathrm{SpO} 2+2.86 \mathrm{NBP}+1.47 \mathrm{UO}-3.15$

2. If HR is low or normal and UO is slightly low or normal, then $y_{2}=833 \mathrm{HR}-294 \mathrm{RR}+939 \mathrm{SpO} 2+2196 \mathrm{NBP}-1499 \mathrm{UO}-2098$

3. If HR is low or normal and UO is slightly low or normal, then $y_{3}=-833 \mathrm{HR}+294 \mathrm{RR}-940 \mathrm{SpO} 2-2199 \mathrm{NBP}+1498 \mathrm{UO}+2102$

4. If $\mathrm{HR}$ is normal or high and $\mathrm{RR}$ is normal or slightly high and $\mathrm{SpO} 2$ is normal and NBP is high and $\mathrm{UO}$ is high,

then $y_{4}=-0.043 \mathrm{HR}+0.142 \mathrm{RR}+0.182 \mathrm{SpO} 2+0.817 \mathrm{NBP}+0.530 \mathrm{UO}-$ 1.21

In order to investigate the interpretability of the model, we applied the normalized values of the inputs that maximize the activation of each rule independently, and looked not only at the output of each rule but also at the final output corresponding to the weighted average of the individual rule outputs. For the model presented in Figure 5, the threshold selected to turn the predicted output into a binary classification was 0.12 , i.e., the classification is 0 if $y<0.12$, and 1 otherwise. For instance, for a low HR, low RR, normal SpO2, slightly high $\mathrm{NBP}$ and low UO, corresponding to $x=[0.2,0.2,0.5,0.7,0.2], y_{1}$ is below 0 and the model output is 0.013 , which translates into a non-necessity of vasopressors intake. On the other hand, if $\mathrm{HR}$ is high and $\mathrm{RR}$ is slightly high and $\mathrm{SpO} 2$ is normal and NBP is high and UO is high, the degree of activation of rule 4 is 1 , and both $y_{4}$ and the model output are above the selected threshold, meaning that the patient will need vasopressors.

\section{Conclusions}

This paper proposes the application of ensemble fuzzy modeling to predict the need of vasopressors administration in septic shock patients. Classification is given by evaluating the relevance of individual classifiers working 
in parallel, where the subspace of each single classifier is defined by nonoverlapping samples for the same feature space, and the final decision is obtained by selecting the best classifier according to different criteria. In contrast to other ensemble techniques, data used to train individual classifiers is obtained by a structured division of samples, based on patient similarities.

The proposed ensemble fuzzy model achieved a significantly higher performance than the single model in the prediction of vasopressors dependence in the ICU, being the a posteriori approach the best performer, with an average AUC of 0.85 . The application of ensemble fuzzy modeling to the benchmark datasets also improves the results of the corresponding single model approach.

The results presented in this paper suggest that using predictive models that deal with patient-specific problems is a more appropriate strategy than using models fitted to the general patient. The proposed ensemble fuzzy model approach can provide prognostic and treatment guidance personalized at the patient level, by only including similar patients in the modeling cohort.

Future work will focus on the implementation of feature selection techniques to select the variables more closely related to the classification problem, in order to improve model performance.

\section{Acknowledgment}

The project was supported by the FCT research project IC4U - Decision support system for preventing ICU readmissions (PTDC/EMS-SIS/3220/2012). This work is funded by Portuguese Funds through the FCT - Foundation for Science and Technology under the project LAETA 2015-2020, reference UID/EMS/50022/2013. S. M. Vieira acknowledges the support by Program Investigador FCT (IF/00833/2014) from FCT, co-funded by the European Social Fund (ESF) through the Operational Program Human Potential (POPH).

[1] S. Herget-Rosenthal, F. Saner, L. S. Chawla, Approach to hemodynamic shock and vasopressors., Clinical Journal of the American Society of Nephrology 3 (2) (2008) 546-53.

[2] W. Zong, M. Saeed, L. Nielsen, A vasopressor advisability index for hemodynamic monitoring., in: Annual Symposium proceedings/AMIA Symposium, 2008, p. 1198. 
[3] G. B. Moody, L. H. Lehman, Predicting acute hypotensive episodes: The 10th annual physionet/computers in cardiology challenge, in: Computers in Cardiology, 2009, IEEE, 2009, pp. 541-544.

[4] F. Cismondi, A. L. Horn, A. S. Fialho, S. M. Vieira, S. R. Reti, J. M. C. Sousa, S. Finkelstein, Multi-stage modeling using fuzzy multi-criteria feature selection to improve survival prediction of icu septic shock patients, Expert Systems with Applications 39 (16) (2012) 12332-12339.

[5] A. S. Fialho, F. Cismondi, S. M. Vieira, J. M. C. Sousa, S. R. Reti, L. A. Celi, M. D. Howell, S. N. Finkelstein, Fuzzy modeling to predict administration of vasopressors in intensive care unit patients, in: 2011 IEEE International Conference on Fuzzy Systems (FUZZ), IEEE, 2011, pp. 2296-2303.

[6] A. S. Fialho, L. A. Celi, F. Cismondi, S. M. Vieira, S. R. Reti, J. M. C. Sousa, S. N. Finkelstein, Disease-based modeling to predict fluid response in intensive care units, Methods of Information in Medicine 52 (6) (2013) 494-502.

[7] K. Strand, H. Flaatten, Severity scoring in the ICU: a review, Acta anaesthesiologica Scandinavica 52 (4) (2008) 467-78.

[8] R. L. Kravitz, N. Duan, J. Braslow, Evidence-based medicine, heterogeneity of treatment effects, and the trouble with averages, The Milbank quarterly 82 (4) (2004) 661-87.

[9] FDA Report. Paving the way for personalized medicine. (2013).

URL http://www.fda.gov/downloads/ScienceResearch/ SpecialTopics/PersonalizedMedicine/UCM372421.pdf

[10] L. A. G. Celi, R. J. Tang, M. C. Villarroel, G. A. Davidzon, W. T. Lester, H. C. Chueh, A Clinical Database-Driven Approach to Decision Support: Predicting Mortality Among Patients with Acute Kidney Injury, Journal of healthcare engineering 2 (1) (2011) 97-110.

[11] M. Berthold, C. Borgelt, F. Höppner, Guide to intelligent data analysis, Vol. 42, Springer, 2010.

[12] T. G. Dietterich, Machine-learning research, AI magazine 18 (4) (1997) 97. 
[13] J. Kittler, M. Hatef, R. P. W. Duin, J. Matas, On combining classifiers, IEEE Transactions on Pattern Analysis and Machine Intelligence 20 (3) (1998) 226-239.

[14] J. Ghosh, Multiclassifier systems: Back to the future, in: Multiple classifier systems, Springer, 2002, pp. 1-15.

[15] A. Sharkey, N. Sharkey, Diversity, selection, and ensembles of artificial neural nets, Neural Networks and their Applications (NEURAP97) (1997) 205-212.

[16] T. G. Dietterich, Ensemble methods in machine learning, in: Multiple classifier systems, Springer, 2000, pp. 1-15.

[17] L. Xiong, A. Y. Shamseldin, K. M. O'connor, A non-linear combination of the forecasts of rainfall-runoff models by the first-order takagi-sugeno fuzzy system, Journal of hydrology 245 (1) (2001) 196-217.

[18] A. P. Weigel, M. A. Liniger, C. Appenzeller, Can multi-model combination really enhance the prediction skill of probabilistic ensemble forecasts?, Quarterly Journal of the Royal Meteorological Society 134 (630) (2008) 241-260.

[19] B. Twala, Multiple classifier application to credit risk assessment, Expert Systems with Applications 37 (4) (2010) 3326-3336.

[20] S. Finlay, Multiple classifier architectures and their application to credit risk assessment, European Journal of Operational Research 210 (2) (2011) 368-378.

[21] L. F. Mendonça, J. M. C. Sousa, J. M. G. Sá da Costa, An architecture for fault detection and isolation based on fuzzy methods, Expert Systems with Applications 36 (2) (2009) 1092-1104.

[22] B. Krawczyk, G. Schaefer, Effective multiple classifier systems for breast thermogram analysis, in: 2012 21st International Conference on Pattern Recognition (ICPR), IEEE, 2012, pp. 3345-3348.

[23] R. Polikar, Ensemble based systems in decision making, IEEE Circuits and Systems Magazine 6 (3) (2006) 21-45. 
[24] K. Woods, W. P. Kegelmeyer Jr, K. Bowyer, Combination of multiple classifiers using local accuracy estimates, IEEE Transactions on Pattern Analysis and Machine Intelligence 19 (4) (1997) 405-410.

[25] T. K. Ho, J. J. Hull, S. N. Srihari, Decision combination in multiple classifier systems, IEEE Transactions on Pattern Analysis and Machine Intelligence 16 (1) (1994) 66-75.

[26] L. Breiman, Bagging predictors, Machine learning 24 (2) (1996) 123140.

[27] Y. Freund, Boosting a weak learning algorithm by majority, in: COLT, Vol. 90, 1990, pp. 202-216.

[28] R. E. Schapire, The strength of weak learnability, Machine learning 5 (2) (1990) 197-227.

[29] L. I. Kuncheva, Clustering-and-selection model for classifier combination, in: Fourth International Conference on Knowledge-Based Intelligent Engineering Systems and Allied Technologies, Vol. 1, IEEE, 2000, pp. $185-188$.

[30] L. Didaci, G. Giacinto, F. Roli, G. L. Marcialis, A study on the performances of dynamic classifier selection based on local accuracy estimation, Pattern Recognition 38 (11) (2005) 2188-2191.

[31] C. Blake, C. J. Merz, UCI repository of machine learning databases.

[32] R. Kruse, C. Döring, M.-J. Lesot, Fundamentals of fuzzy clustering, Advances in fuzzy clustering and its applications (2007) 3-30.

[33] J. C. Dunn, A fuzzy relative of the isodata process and its use in detecting compact well-separated clusters, Journal of Cybernetics 3 (3) (1973) $32-57$.

[34] J. C. Bezdek, Pattern Recognition with Fuzzy Objective Function Algorithms, Kluwer Academic Publishers, Norwell, MA, USA, 1981.

[35] X. L. Xie, G. Beni, A validity measure for fuzzy clustering, IEEE Transactions on Pattern Analysis and Machine Intelligence 13 (8) (1991) 841847. 
[36] A. M. Bensaid, L. O. Hall, J. C. Bezdek, L. P. Clarke, M. L. Silbiger, J. A. Arrington, R. F. Murtagh, Validity-guided (re) clustering with applications to image segmentation, IEEE Transactions on Fuzzy Systems 4 (2) (1996) 112-123.

[37] N. R. Pal, J. C. Bezdek, On cluster validity for the fuzzy c-means model, IEEE Transactions on Fuzzy Systems 3 (3) (1995) 370-379.

[38] B. Balasko, J. Abonyi, B. Feil, Fuzzy clustering and data analysis toolbox - for use with matlab, Department of Process Engineering, University of Veszprem, Veszprem.

[39] M. Sugeno, T. Yasukawa, A fuzzy-logic-based approach to qualitative modeling, IEEE Transactions on Fuzzy Systems 1 (1) (1993) 7-31.

[40] T. Takagi, M. Sugeno, Fuzzy identification of systems and its applications to modeling and control, IEEE Transactions on Systems, Man and Cybernetics (1) (1985) 116-132.

[41] J. M. C. Sousa, U. Kaymak, Fuzzy decision making in modeling and control, Vol. 27, World Scientific Singapore, 2002.

[42] J. A. Swets, Measuring the accuracy of diagnostic systems, Science 240 (4857) (1988) 1285-1293.

[43] M. Grzybowski, J. G. Younger, Statistical methodology: III. receiver operating characteristic ROC curves, Academic emergency medicine 4 (8) (1997) 818-826.

[44] T. Fawcett, An introduction to ROC analysis, Pattern Recogn. Lett. 27 (8) (2006) 861-874.

[45] M. Saeed, M. Villarroel, A. T. Reisner, G. Clifford, L.-W. Lehman, G. Moody, T. Heldt, T. H. Kyaw, B. Moody, R. G. Mark, Multiparameter intelligent monitoring in intensive care ii (mimic-ii): A public-access intensive care unit database, Critical Care Medicine 39 (2011) 952-960.

[46] G. D. Clifford, W. J. Long, G. B. Moody, P. Szolovits, Robust parameter extraction for decision support using multimodal intensive care data, Philosophical Transactions of the Royal Society A: Mathematical, Physical and Engineering Sciences 367 (1887) (2009) 411-429. 
[47] P. D. Allison, Missing Data, no. 136 in Quantitative Applications in the Social Sciences, SAGE Publications, 2001.

[48] A. S. Fialho, F. Cismondi, S. M. Vieira, J. M. C. Sousa, S. R. Reti, R. Welsh, Welsch, M. D. Howel, S. N. Finkelstein, Missing data in large intensive care units databases, Critical Care Medicine 38(12):U6-U6.

[49] D. C. Hoaglin, F. Mosteller, J. W. Tukey, Understanding robust and exploratory data analysis, Wiley Classics Library Editions, Wiley, 2000.

[50] F. Cismondi, A. S. Fialho, S. M. Vieira, J. M. C. Sousa, S. R. Reti, M. D. Howell, S. N. Finkelstein, Computational intelligence methods for processing misaligned, unevenly sampled time series containing missing data, in: 2011 IEEE Symposium on Computational Intelligence and Data Mining (CIDM), 2011, pp. 224-231.

[51] F. Cismondi, A. S. Fialho, S. M. Vieira, S. R. Reti, J. M. C. Sousa, S. N. Finkelstein, Missing data in medical databases: Impute, delete or classify?, Artificial intelligence in medicine 58 (1) (2013) 63-72.

[52] C.-W. Hsu, C.-J. Lin, A comparison of methods for multiclass support vector machines, Neural Networks, IEEE Transactions on 13 (2) (2002) 415-425.

[53] M. Skurichina, L. I. Kuncheva, R. P. Duin, Bagging and boosting for the nearest mean classifier: Effects of sample size on diversity and accuracy, in: Multiple Classifier Systems, Springer, 2002, pp. 62-71.

[54] K. P. Bennett, J. A. Blue, A support vector machine approach to decision trees, in: Neural Networks Proceedings, 1998. IEEE World Congress on Computational Intelligence. The 1998 IEEE International Joint Conference on, Vol. 3, IEEE, 1998, pp. 2396-2401.

[55] K. Polat, S. Şahan, H. Kodaz, S. Güneş, Breast cancer and liver disorders classification using artificial immune recognition system (airs) with performance evaluation by fuzzy resource allocation mechanism, Expert Systems with Applications 32 (1) (2007) 172-183.

[56] P. J. Tan, D. L. Dowe, Mml inference of oblique decision trees, in: AI 2004: Advances in Artificial Intelligence, Springer, 2005, pp. 1082-1088. 
[57] F. Cao, H. Ye, D. Wang, A probabilistic learning algorithm for robust modeling using neural networks with random weights, Information Sciences 313 (2015) 62-78.

[58] I. Anagnostopoulos, C. Anagnostopoulos, D. Vergados, A. Rouskas, G. Kormentzas, The wisconsin breast cancer problem: Diagnosis and ttr/dfs time prognosis using probabilistic and generalised regression information classifiers, Oncology reports 15 (4) (2006) 975-981.

[59] R. Babuška, Fuzzy modelling and identification toolbox, Control Engineering Laboratory, Faculty of Information Technology and Systems, Delft University of Technology, Delft, The Netherlands, version 3. 